\title{
Motion Freeze for Respiration Motion Correction in PET/CT: A Preliminary Investigation with Lung Cancer Patient Data
}

\author{
Tzung-Chi Huang, ${ }^{1,2}$ Kuei-Ting Chou, ${ }^{1}$ Yao-Ching Wang, ${ }^{3}$ and Geoffrey Zhang ${ }^{4}$ \\ ${ }^{1}$ Department of Biomedical Imaging and Radiological Science, China Medical University, 91 Hsueh-Shih Road, Taichung City, Taiwan \\ ${ }^{2}$ Department of Biomedical Informatics, Asia University, Taichung City, Taiwan \\ ${ }^{3}$ Department of Radiation Oncology, China Medical University Hospital, Taichung City, Taiwan \\ ${ }^{4}$ Department of Radiation Oncology, Moffitt Cancer Center, Tampa, FL, USA
}

Correspondence should be addressed to Tzung-Chi Huang; tzungchi.huang@mail.cmu.edu.tw

Received 6 May 2014; Revised 13 August 2014; Accepted 16 August 2014; Published 28 August 2014

Academic Editor: Wei Lu

Copyright (C) 2014 Tzung-Chi Huang et al. This is an open access article distributed under the Creative Commons Attribution License, which permits unrestricted use, distribution, and reproduction in any medium, provided the original work is properly cited.

Purpose. Respiratory motion presents significant challenges for accurate PET/CT. It often introduces apparent increase of lesion size, reduction of measured standardized uptake value (SUV), and the mismatch in PET/CT fusion images. In this study, we developed the motion freeze method to use $100 \%$ of the counts collected by recombining the counts acquired from all phases of gated PET data into a single 3D PET data, with correction of respiration by deformable image registration. Methods. Six patients with diagnosis of lung cancer confirmed by oncologists were recruited. PET/CT scans were performed with Discovery STE system. The 4D PET/CT with the Varian real-time position management for respiratory motion tracking was followed by a clinical 3D PET/CT scan procedure in the static mode. Motion freeze applies the deformation matrices calculated by optical flow method to generate a single 3D effective PET image using the data from all the 4D PET phases. Results. The increase in SUV and decrease in tumor size with motion freeze for all lesions compared to the results from 3D and 4D was observed in the preliminary data of lung cancer patients. In addition, motion freeze substantially reduced tumor mismatch between the CT image and the corresponding PET images. Conclusion. Motion freeze integrating $100 \%$ of the PET counts has the potential to eliminate the influences induced by respiratory motion in PET data.

\section{Introduction}

Positron emission tomography/computed tomography (PET/CT) combining with the functional F-18 fluoro-2deoxyglucose $\left({ }^{18} \mathrm{~F}\right.$-FDG) PET scan and the anatomical CT scan has become an essential modality for detecting tumors, planning radiation treatment, and evaluating response to therapy [1-3]. However, the respiratory motion during PET acquisition leads to artifacts in PET/CT. As the CT acquisition is much faster (in seconds) than PET scan (in minutes), CT image represents an almost instantaneous snapshot in comparison to the averaged PET images. Consequently, CT data is not always in spatial correspondence with the PET data and CT applied for attenuation correction (AC) in PET may lead to the underestimation of $\mathrm{SUV}_{\max }$ of tumors, overestimation of tumor volume, and mismatched PET and CT images and tumor inaccurate localization, hence potential misdiagnoses [4-8].

Several methods have been investigated mostly to manage the respiratory motion problem. These methods include breathing instruction, respiratory-gate (4D) PET/CT, motion-corrected PET reconstruction, and postprocessing methods [9-15]. Breathing instruction methods like deepinspiration breath-hold (DIBH) during the CT scan reduce the occurrence and the severity of respiratory curvilinear artifacts on coregistered PET/CT images. A study on 108 patients showed that the $S U V_{\max }$ increased as much as $51.8 \%$ on average from free-breathing PET to DIBH PET for lesions in the lower lung region as reported by Kawano et al. [9]. De Juan et al. reported that PET reconstructed images from patients of normal end-expiration breath-hold group 
TABLE 1: Clinical characteristics of study subjects and tumors.

\begin{tabular}{lccccc}
\hline Patient \# & Sex & Age $(\mathrm{y})$ & Histology & Location & Type \\
\hline 1 & M & 64 & NSCLC & Right lower lobe & Lymph node \\
2 & M & 59 & NSCLC & Left lower lobe & Lymph node \\
3 & M & 63 & NSCLC & Right lower lobe & Lymph node \\
4 & F & 57 & NSCLC & Left upper lobe & Lymph node \\
5 & M & 61 & NSCLC & Right lower lobe & Lymph node \\
6 & M & 64 & NSCLC & Left lower lobe & Lymph node \\
\hline
\end{tabular}

${ }^{*}$ NSCLC $=$ Non-small-cell lung carcinoma.

had $28 \%$ fewer artifacts as compared with imaging by free breathing imaging [10]. These breathing instruction methods are not feasible for patients with limited pulmonary function to control their breathing. Nehmeh et al. spatially matched the 4D CT data with the gated PET images according to the externally monitored breathing signal and showed improved lesion registration [11]. Cine average CT was developed for $\mathrm{AC}$ in PET and showed significantly less severe misalignments and artifacts as compared with conventional helical CT (HCT) based AC [12]. The main problem of 4D technology is relatively high radiation dose compared to conventional HCT. With combining gated CT and imaging processing, the interpolated average CT used for PET/CT AC corrected the PET/CT misregistration and enhanced lesion quantitation accompanied with radiation dose reduction was proposed by Huang et al. $[13,14]$. However averaging caused the lack of total effective counts in PET data. Other studies using motion estimation into the iterative reconstruction process lowered image noise of a gated PET frame but utilized lower percentage of the total PET counts [15].

Previous studies waste counts in PET images acquisition for respiratory motion reduction. In this study, we proposed the motion freeze concept to use $100 \%$ of the counts collected by recombining $100 \%$ of the counts acquired from all phases of gated PET data into a single 3D PET data, with correction of respiration by deformable image registration on the 4D CT images.

\section{Material and Methods}

2.1. Data Acquisition. The current study was conducted from November to December 2013. Six patients with diagnosis of lung cancer confirmed by oncologists were recruited. The summary of the clinical characteristics of the patients is shown in Table 1 . The patients were all injected with $370 \mathrm{MBq}$ of ${ }^{18} \mathrm{~F}$-FDG. During the uptake phase that lasted for approximately 40 minutes, the patients remained in a still position. The first whole-body image was obtained when the patients were in a supine position and the acquisition time per bed position was $1.5 \mathrm{~min}$. Free-breathing whole-body CT was conducted at $120 \mathrm{kV}$ in helical mode (HCT) with a smart mA (range 30-210 mA), 1.375: 1 pitch, and 0.5 s gantry rotation. For the thoracic PET, the patients were scanned for two bed positions with $1.5 \mathrm{~min} / \mathrm{bed}$. The transaxial field-ofviews (FOVs) for PET and CT were set on 70 and $50 \mathrm{~cm}$, respectively.
PET/CT scans were performed with Discovery STE system (GE Healthcare, Milwaukee, WI, USA). The 4D PET/CT with the Varian real-time position management (RPM system, Varian Medical Systems, Inc. Palo Alto, CA) for respiratory motion tracking was followed by a clinical 3D PET/CT-HCT scan procedure in the static mode. During all studies, the patients remained in the same position. 4D PET/CT data were acquired into 10 discrete bins in synchronization with the breathing cycle using phase gating mode [11]. 4D PET/CT protocol consisted in a $4 \mathrm{D}$ CT scan over the thoracic region. The $4 \mathrm{D}$ CT data was performed by using a step-and-shoot cine mode acquisition technique and the sinograms were reconstructed on a $512 \times 512$ image matrix. The pixel size in the transaxial slice of the 4D CT images was approximately $0.98 \times 0.98 \mathrm{~mm}^{2}$, and the slice thickness was $2.5 \mathrm{~mm}$. In $4 \mathrm{D} \mathrm{CT}$, the 10 respiratory phases were labeled as T5\%, T15\%,.., T95\%, with the T5\% phase corresponding approximately to the normal end-inspiration and the T55\% to the end expiration. After the termination of the 4D CT scan, a 4D PET scan of two PET FOVs centered over the same anatomical region was acquired in the gated mode. 4D-PET data were corrected for attenuation with $4 \mathrm{D}$ CT images, then with respiratory phase matched cine CT images [16]. 4D PET images were reconstructed on a $128 \times 128$ image matrix with a voxel size of $4.46 \times 5.46 \times$ $3.27 \mathrm{~mm}^{3}$. The PET images were linearly interpolated to the resolution and geometry of the CT scan for fusion image. The ordered subset expectation maximization reconstruction method was used with 2 iterations and 20 subsets and the postreconstruction Gaussian filter was applied with a fullwidth at half-maximum of $7 \mathrm{~mm}$. Written informed consent was obtained from all patients, and the collection of clinical patient data was approved by the ethics committees of the clinical institution.

2.2. Motion Freeze. The purpose of motion freeze is to apply the deformation matrix calculated by optical flow method (OFM) [17], one of deformable image registration methods, to generate a single $3 \mathrm{D}$ effective PET image using the total $100 \%$ counts from different gates. Two new features including the multiloop calculation and the multiresolution to enhance the accuracy of registration were implanted into OFM [18]. The modified OFM based on image intensity gradient was utilized for deformable image registration and the accuracy of registration was reported in previous studies [18-20]. The deformable registration accuracy in the presented patient 
TABLE 2: Summary of different quantitative results for 3D, 4D, and motion freeze methods.

\begin{tabular}{|c|c|c|c|c|c|c|c|c|c|}
\hline \multirow{2}{*}{ Patient number } & \multicolumn{3}{|c|}{$\mathrm{SUV}_{\text {mean }}$} & \multicolumn{3}{|c|}{$\mathrm{SUV}_{\max }$} & \multicolumn{3}{|c|}{ Tumor Volume $\left(\mathrm{cm}^{3}\right)$} \\
\hline & $\mathrm{PET}_{3 \mathrm{D}}$ & $\mathrm{PET}_{4 \mathrm{D}}$ & $\mathrm{PET}_{\mathrm{MF}}$ & $\mathrm{PET}_{3 \mathrm{D}}$ & $\mathrm{PET}_{4 \mathrm{D}}$ & $\mathrm{PET}_{\mathrm{MF}}$ & $\mathrm{PET}_{3 \mathrm{D}}$ & $\mathrm{PET}_{4 \mathrm{D}}$ & $\mathrm{PET}_{\mathrm{MF}}$ \\
\hline 1 & 7.4 & 7.5 & 7.6 & 13.5 & 13.7 & 13.9 & 68.2 & 51.1 & 50.2 \\
\hline 2 & 7.7 & 7.9 & 7.9 & 13.2 & 15.3 & 15.8 & 173.1 & 144.7 & 140.0 \\
\hline 3 & 6.6 & 8.2 & 8.4 & 12.4 & 14.8 & 16.3 & 120.0 & 109.4 & 100.8 \\
\hline 4 & 2.6 & 3.3 & 4.7 & 9.9 & 9.9 & 10.0 & 22.0 & 14.7 & 12.7 \\
\hline 5 & 1.8 & 1.8 & 2.1 & 8.2 & 9.3 & 9.8 & 132.2 & 128.0 & 123.1 \\
\hline 6 & 3.0 & 3.8 & 4.4 & 14.8 & 15.9 & 15.9 & 42.2 & 34.8 & 27.6 \\
\hline
\end{tabular}

cohort is validated using manual annotation. In this study, the T55\% (end expiration) phase, which is the most reproducible phase in the respiration cycle, was selected as the reference phase. The elements of deformable image registration matrix are three-dimensional vectors with magnitudes relating the computed displacement of voxels from the image of interest to the reference image. Once this deformation matrix has been calculated, the matrix can be applied to map different phases of 4D PET images to the reference phase of PET. Finally, each SUV of the corresponding voxel in the resulted 3D PET image, $\mathrm{PET}_{\mathrm{MF}}$, was determined by the statistic median process, in which $100 \%$ of counts from all the phases was included. The flow diagram of the motion freeze which CT derived motion fields are used to correct PET was shown in Figure 1.

2.3. Quantitative Analysis. The PET data acquired from conventional static PET/HCT technology were denoted as $\mathrm{PET}_{3 \mathrm{D}}$. In the PET images, a 3D volume of interest (VOI) was automatically drawn. The lower threshold was set to $42 \%$ of the maximum activity concentration value within the lesion [21]. The mean SUV ( $\mathrm{SUV}_{\text {mean }}$ ) and maximum SUV $\left(\mathrm{SUV}_{\max }\right)$ in the VOI were calculated. The corresponding delineation for VOI in the CT images was conducted by an experienced radiation oncologist. The 4D PET phase with the highest $\mathrm{SUV}_{\max }$ (best bin) was selected as $\mathrm{PET}_{4 \mathrm{D}}$ for evaluation. The rebinned PET image by motion freeze approach was defined as $\mathrm{PET}_{\mathrm{MF}}$. Quantitative analysis was performed by comparing the $\mathrm{SUV}_{\text {mean }}, \mathrm{SUV}_{\text {max }}$, and tumor volume and the percentage difference (PD) was calculated using $\mathrm{PET}_{3 \mathrm{D}}$ as reference. The coordinates of the centroid of the lesion displayed in the PET and corresponding CT images were determined based on the delineated VOIs. The distance " $d$ " between the lesion centroid identified using PET and the one in the associated CT image was then measured. Tumor deformation (TD) between the end-inspiration (T5\%) and the end-inspiration (T55\%) from 4D-CT data was also calculated using the average of displacements in three directions: the anterior-posterior (AP), lateral (LAT), and superior-inferior (SI) [22].

\section{Results}

An example (Patient number 1) of deformable image registration using the 3D optical flow method to map the T5\% PET to T55\% PET is shown in Figure 2. For Patient number 4, as demonstrated in Figure 3, the lesion in the left upper lobe was substantially improved for $\mathrm{PET}_{4 \mathrm{D}}$ and $\mathrm{PET}_{\mathrm{MF}}$ as compared to $\mathrm{PET}_{3 \mathrm{D}}$, with increase of $\mathrm{SUV}_{\text {mean }}$ of $27 \%$ and $81 \%$, respectively. The identification of the PET images was also improved for $\mathrm{PET}_{\mathrm{MF}}$ as compared to $\mathrm{PET}_{3 \mathrm{D}}$ in this patient as indicated in the line profiles drawn on Figure 3(d), where the blurring image appeared to be smaller with improved structure details in the outside of lesion on $\mathrm{PET}_{\mathrm{MF}}$. The $\mathrm{PET}_{3 \mathrm{D}}$ had severe cold artifacts around the left diaphragm region, while the artifacts were reduced in the $\mathrm{PET}_{\mathrm{MF}}$.

The $S U V_{\text {mean }}, S V_{\text {max }}$, and tumor volume for all patients are summarized in Table 2. Compared to $\mathrm{PET}_{3 \mathrm{D}}$ and $\mathrm{PET}_{4 \mathrm{D}}$, the $\mathrm{SUV}_{\text {mean }}$ in $\mathrm{PET}_{\mathrm{MF}}$ was higher for all the tumors. The average increase of PDs of SUV mean for all patients was $14 \%$ and $22 \%$ for $\mathrm{PET}_{4 \mathrm{D}}$ and $\mathrm{PET}_{\mathrm{MF}}$ as compared to $\mathrm{PET}_{3 \mathrm{D}}$, respectively. The tumor volumes measured in $\mathrm{PET}_{\mathrm{MF}}$ were significantly smaller than the values measured in $\mathrm{PET}_{3 \mathrm{D}}$ and $\mathrm{PET}_{4 \mathrm{D}}$. The PDs of the tumor volume measured in $\mathrm{PET}_{\mathrm{MF}}$ compared to $\mathrm{PET}_{3 \mathrm{D}}$ were in the range between $17 \%$ and $19 \%$. The values of $\mathrm{SUV}_{\max }$ were all increased by $\mathrm{PET}_{\mathrm{MF}}$ as compared of $\mathrm{PET}_{3 \mathrm{D}}$.

Estimates of TD between the normal end-inspiration and end-expiration phases and the differences in the centroid of the tumors $(d)$ are represented in Table $3 . \mathrm{PET}_{\mathrm{MF}} / \mathrm{CT}_{\mathrm{T} 55 \%}$ fusion image substantially reduced tumor mismatch $d$ between the CT image and the corresponding PET images, as shown in Table 3, with an average decrease of $6.1 \mathrm{~mm}$ among all of the lesions as compared to 3D-PET/HCT, whereas an average decrease of $4.3 \mathrm{~mm}$ was observed when using $4 \mathrm{D}$ PET/CT (best-bin).

\section{Discussion}

The idea of motion freeze is conceived by GE Q. Freeze technology (GE Healthcare, Milwaukee, WI, USA) is the latest commercial product coping with motion correction for PET/CT. Q. Freeze technology registering PET by OFM was applied for motion estimation, whereas the validation with ground truth was missing and only the relative correlation coefficient was presented [23]. The OFM basically calculates the flow information according to the changes in image intensity between two images. The image resolution is generally coarse in PET image and also the structure information was not represented in PET image. Consequently, the precise registration in PET image was difficult to achieve. 


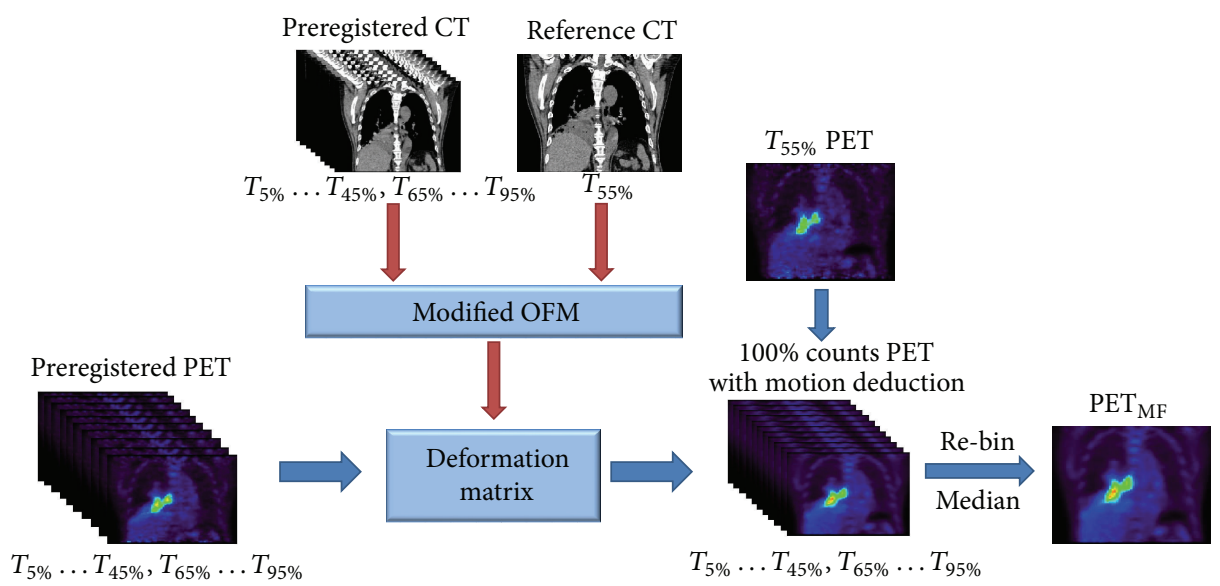

FIGURE 1: Flow diagram of the motion freeze process used in this study.

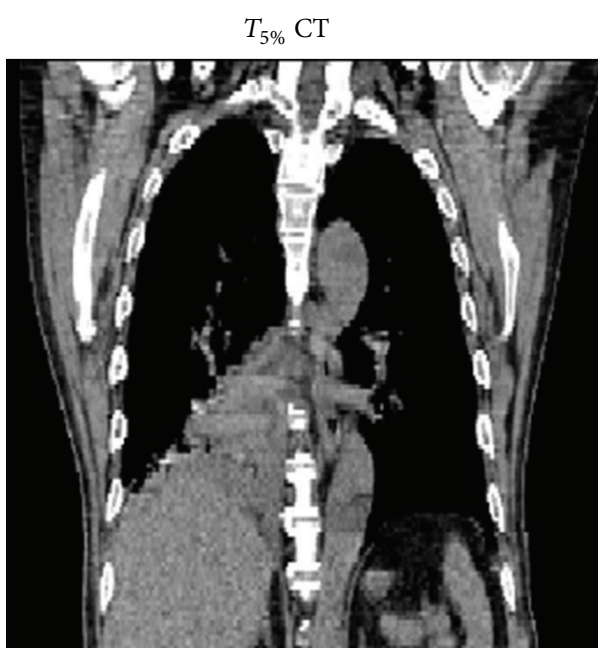

(a)

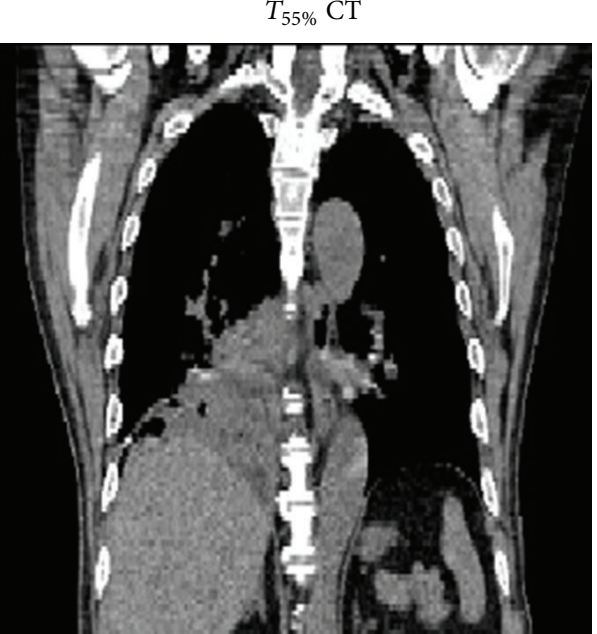

(b)

Deformation vector

$T_{5 \%}$ PET

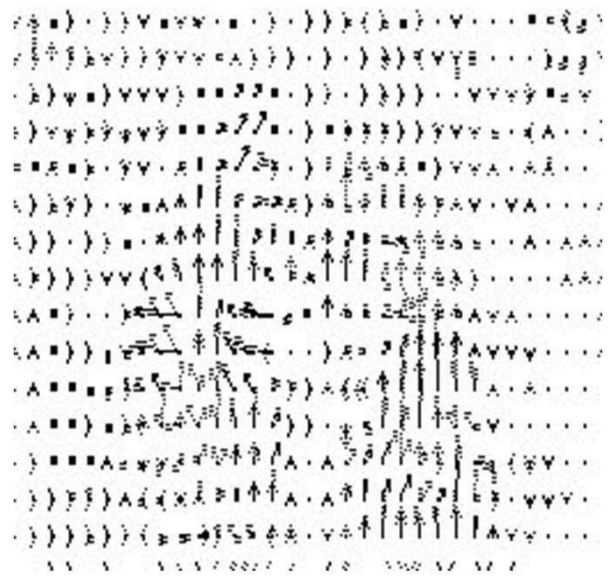

(c)

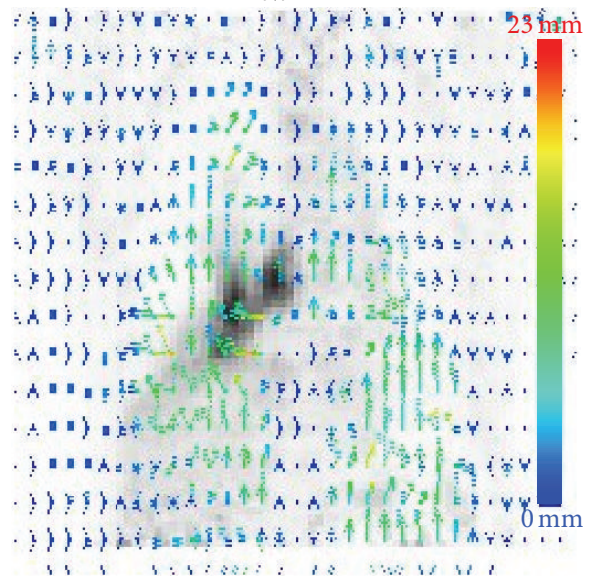

(d)

Figure 2: Example of deformable image registration using the 3D optical flow method to map the T5\% PET to T55\% PET. (a) A coronal view of the normal end-inspiration CT (T5\%, original image) of 4D-CT. (b) The corresponding slice of the location in (a) at end-expiration CT (T55\%, reference image) of 4D CT. (c) The calculated deformation vectors for the registration of original image to the reference. (d) The corresponding T5\% PET with the 3D OFM deformation vectors superimposed. 

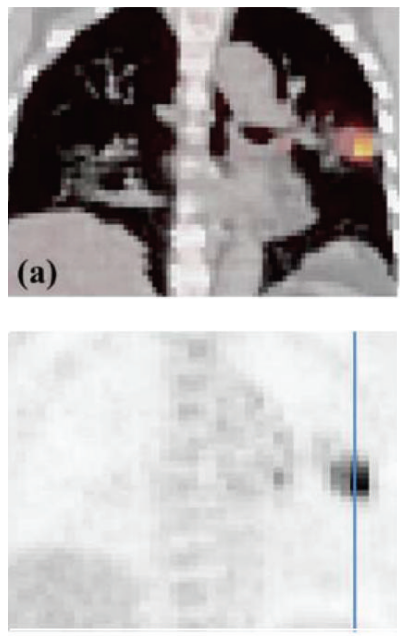

(a)
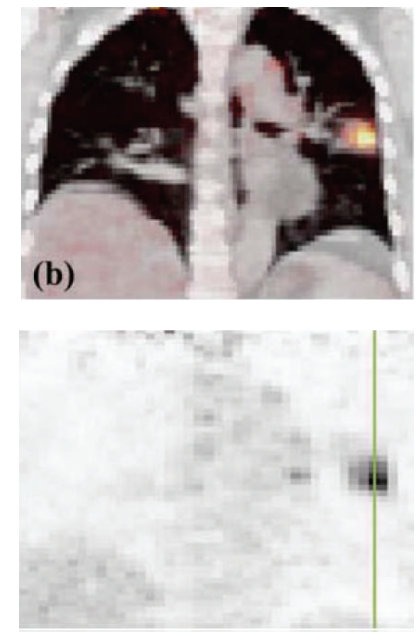

(b)
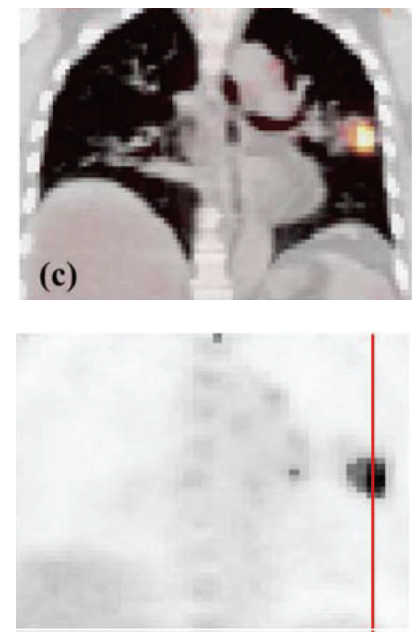

(c)

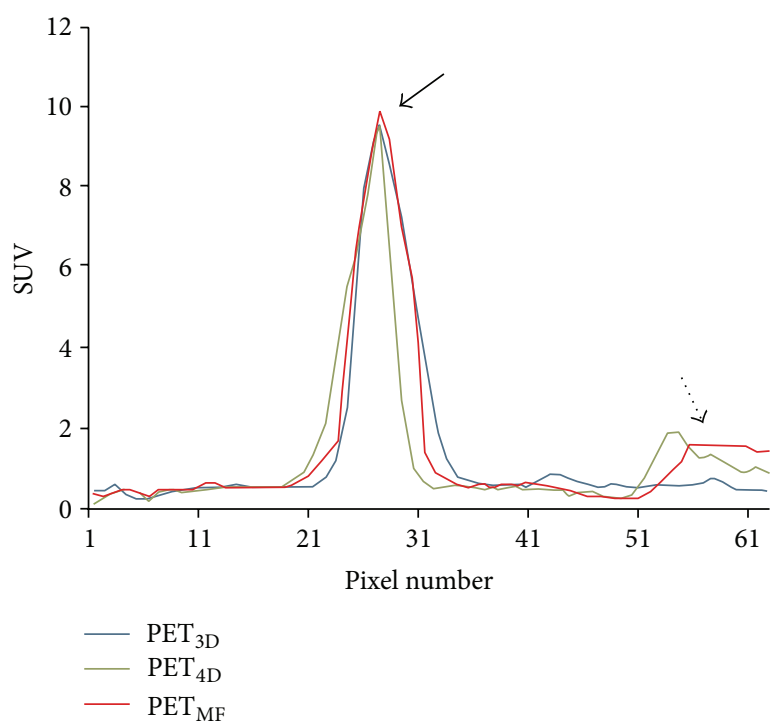

(d)

FIgURE 3: The coronal views of the fused PET/CT images from (a) 3D, (b) 4D (best bin), (c) motion freeze technology for patient number 4. A lesion in the left upper lobe of the lung and artifacts around the right diaphragm were observed on the 3D HCT images, and (d) vertical image profiles drawn across the lesion on $\mathrm{PET}_{3 \mathrm{D}}, \mathrm{PET}_{4 \mathrm{D}}$, and $\mathrm{PET}_{\mathrm{MF}}$ images. Black arrow indicated the lesion area and the dotted black arrow indicated the diaphragm region.

TABLE 3: Summary of tumor motion estimations and differences of centroid of the tumors.

\begin{tabular}{|c|c|c|c|c|c|c|c|}
\hline \multirow{2}{*}{ Patient number } & \multicolumn{4}{|c|}{ Deformation of tumor motion (mm) } & \multicolumn{3}{|c|}{$d(\mathrm{~mm})$} \\
\hline & AP & LAT & SI & TD & 3D-PET/HCT & 4D-PET/CT & $\mathrm{PET}_{\mathrm{MF}} / \mathrm{CT}_{\mathrm{T} 55 \%}$ \\
\hline 1 & $1.1 \pm 1.0$ & $0.9 \pm 0.9$ & $7.3 \pm 2.6$ & $7.7 \pm 2.6$ & 22.7 & 16.4 & 11.4 \\
\hline 2 & $1.9 \pm 1.2$ & $1.4 \pm 1.0$ & $3.1 \pm 1.4$ & $4.4 \pm 1.1$ & 6.1 & 5.5 & 4.6 \\
\hline 3 & $0.9 \pm 1.2$ & $0.5 \pm 0.5$ & $2.5 \pm 1.9$ & $2.88 \pm 2.3$ & 15.5 & 10.5 & 10.4 \\
\hline 4 & $0.6 \pm 0.5$ & $0.9 \pm 1$ & $2.6 \pm 1$ & $3.0 \pm 0.8$ & 7.1 & 3.1 & 1.4 \\
\hline 5 & $3.2 \pm 2.2$ & $2.5 \pm 2.2$ & $3.6 \pm 1.9$ & $5.9 \pm 2.6$ & 13.5 & 6.1 & 5.7 \\
\hline 6 & $3.6 \pm 2.5$ & $1.2 \pm 0.8$ & $8.5 \pm 5.4$ & $9.6 \pm 2.1$ & 7.7 & 5.2 & 2.3 \\
\hline
\end{tabular}


The 4D CT images were used for registration using OFM in this study. The validations of the proposed OFM were reported in previous phantom and clinical patient studies [18-20]. In OFM for deformable image registration, CT as image modality is superior to PET. Although model tissue viscosity and elasticity are used to take into consideration the anatomical environment after OFM registration in GE Q.Freeze technology, the model should not be identical for all patients and usually not feasible for patients with lung injuries [24].

Respiratory motion will blur the activity image and hence reduce the measured activity concentration and corresponding SUV. Motion freeze registers all the gated phases to the selected gated phase (T55\%) and the 100\% counts are all used to provide the best quality of PET image with motion deduction. The quantitation recovery on both the SUV derived from the motion freeze process as compared with the SUV obtained from the corresponding static PET was observed in our results. The visual blur in the static image resulting from respiratory motion generally appears stretched along the direction of the motion and can be quantified by the tumor volume. The estimate of the tumor volume obtained from a static image is usually higher than that for individual gates. The blur reduction was observed by the comparison between the tumor volumes obtained from the motion freeze processing and that from the corresponding static (3D) image.

The improvement of SUV $V_{\max }$ in 4D-PET and $\mathrm{PET}_{\mathrm{MF}}$ was not improved substantially as compared to $3 \mathrm{D}$-PET in Patient 4. In this case, the tumor was in the left upper lobe (Table 1) thus the respiratory motion was relative small (Table 3). Because of the small motion, the blurring effect on $\mathrm{SUV}_{\max }$ in $3 \mathrm{D}$ PET is relatively smaller. Besides, the 3D PET was obtained 21 minutes later than the $4 \mathrm{D}$ PET and thus the uptake in the tumor could be larger at the time when the 3D PET was taken. Similar cases were reported in another study [22]. Even in the case with little improvement of $S U V_{\text {max }}$ represented in 4D PET, motion freeze can improve the quantification of counts for such the case too. After this feasibility study, we may conduct a detailed study with more patients' data to quantitatively investigate how the motion size affects the $S U V_{\max }$ using this method.

Because the motion freeze resulted from 4D PET/CT imaging, different gating techniques or schemes in $4 \mathrm{D}$ PET/CT imaging could affect the motion freeze outcomes $[16,25,26]$. The effects of the gating schemes from $4 \mathrm{D}-\mathrm{CT}$ for $\mathrm{AC}$ applied on $\mathrm{PET}_{\mathrm{MF}}$ effectiveness are being further investigated in our ongoing study.

The major limitations to widespread clinical adoption of respiratory motion correction are the need to use extra CT dose and the need for additional acquisition time. Motion freeze technology combining the interpolated CT technology has high potential to overcome these challenges by using the entire acquired data to create a single $3 \mathrm{D}$ motion corrected image and to provide quantitative accuracy equivalent to 4D PET/CT with shorter acquisition times and less dose reduction $[13,14]$. Unlike conventional 4D PET imaging, motion freeze technology combines $100 \%$ of the PET counts into a $3 \mathrm{D}$ motion corrected image that has a comparable acquisition time and the equivalent image noise of a static acquisition. With aids of the motion freeze technology, the acquisition of each bed could decrease and still keep the high counts for PET image. In radiation dose reduction from the $4 \mathrm{D}$-CT, the equivalent $4 \mathrm{D}$ phase-matched $\mathrm{PET} / \mathrm{CT}$ could be done by incorporation of active breathing controller and the interpolated CT (ICT) method used for PET/CT attenuation correction mismatch [27]. The resulting image has advantages in frozen respiratory motion, increased SUV, less acquisition time, and CT dose reduction.

For patients who have serious irregular breathing amplitude/pattern which may cause breathing pattern change between CT and PET scans, this method may suffer some uncertainty. In 4D PET/CT, 4D-CT is often used for PET image attenuation correction. For patients who cannot regularly breathe, artifacts are likely in the reconstructed $4 \mathrm{D}$ CT. If breathing amplitude/pattern is changed between CT and PET scans, the 4D-PET data would not be accurate after attenuation correction. Any postscan image processing would be consequently affected. After the CT deformation matrices, which are possibly generated by a different breathing amplitude, are applied to the PET data, the integrated PET may not be exactly reflecting the reality.

\section{Conclusion}

In this study, the motion freeze technology was developed and PET acquisitions were corrected for respiratory motion, leading to superior image quality and increased quantitative SUV in comparison to conventional static imaging. Motion freeze also reduced the lesion volume and lesion maximum activity errors compared to conventional static PET imaging. Motion freeze integrating $100 \%$ of the PET counts has the potential to eliminate the influences induced by respiratory motion in PET data.

\section{Conflict of Interests}

The authors declare that they have no conflict of interest.

\section{Acknowledgments}

This study was financially supported by the National Science Council of Taiwan (NSC-102-2221-E-039-010-MY3) and by the school project of China Medical University (CMU102BC-1).

\section{References}

[1] D. Lardinois, W. Weder, T. F. Hany et al., "Staging of non-smallcell lung cancer with integrated positron-emission tomography and computed tomography," The New England Journal of Medicine, vol. 348, no. 25, pp. 2500-2507, 2003.

[2] R. L. Wahl, H. Jacene, Y. Kasamon, and M. A. Lodge, "From RECIST to PERCIST: evolving considerations for PET response criteria in solid tumors," Journal of Nuclear Medicine, vol. 50, no. $1,2009$.

[3] M. MacManus, U. Nestle, K. E. Rosenzweig et al., "Use of PET and PET/CT for radiation therapy planning: IAEA expert report 
2006-2007," Radiotherapy and Oncology, vol. 91, no. 1, pp. 85-94, 2009.

[4] S. A. Nehmeh and Y. E. Erdi, "Respiratory motion in positron emission tomography/computed tomography: a review," Seminars in Nuclear Medicine, vol. 38, no. 3, pp. 167-176, 2008.

[5] S. J. Park, D. Ionascu, J. Killoran et al., "Evaluation of the combined effects of target size, respiratory motion and background activity on 3D and 4D PET/CT images," Physics in Medicine and Biology, vol. 53, no. 13, pp. 3661-3679, 2008.

[6] U. Nestle, S. Kremp, A. Schaefer-Schuler et al., "Comparison of different methods for delineation of18F-FDG PET-positive tissue for target volume definition in radiotherapy of patients with non-small cell lung cancer," Journal of Nuclear Medicine, vol. 46, no. 8, pp. 1342-1348, 2005.

[7] M. M. Osman, C. Cohade, Y. Nakamoto, and R. L. Wahl, "Respiratory motion artifacts on PET emission images obtained using CT attenuation correction on PET-CT,' European Journal of Nuclear Medicine and Molecular Imaging, vol. 30, no. 4, pp. 603-606, 2003.

[8] C. Liu, L. A. Pierce II, A. M. Alessio, and P. E. Kinahan, "The impact of respiratory motion on tumor quantification and delineation in static PET/CT imaging," Physics in Medicine and Biology, vol. 54, no. 24, pp. 7345-7362, 2009.

[9] T. Kawano, E. Ohtake, and T. Inoue, "Deep-inspiration breathhold PET/CT of lung cancer: maximum standardized uptake value analysis of 108 patients," Journal of Nuclear Medicine, vol. 49, no. 8, pp. 1223-1231, 2008.

[10] R. de Juan, B. Seifert, T. Berthold, G. K. von Schulthess, and G. W. Goerres, "Clinical evaluation of a breathing protocol for PET/CT," European Radiology, vol. 14, no. 6, pp. 1118-1123, 2004.

[11] S. A. Nehmeh, Y. E. Erdi, T. Pan et al., "Four-dimensional (4D) PET/CT imaging of the thorax," Medical Physics, vol. 31, no. 12, pp. 3179-3186, 2004.

[12] T. Pan, O. Mawlawi, S. A. Nehmeh et al., "Attenuation correction of PET images with respiration-averaged CT images in PET/CT,' Journal of Nuclear Medicine, vol. 46, no. 9, pp. 14811487, 2005.

[13] T. C. Huang, G. S. P. Mok, S. J. Wang, T. H. Wu, and G. Zhang, "Attenuation correction of PET images with interpolated average CT for thoracic tumors," Physics in Medicine and Biology, vol. 56, no. 8, pp. 2559-2567, 2011.

[14] G. S. P. Mok, T. Sun, T.-C. Huang, and M. I. Vai, "Interpolated average CT for attenuation correction in PET-A simulation study," IEEE Transactions on Biomedical Engineering, vol. 60, no. 7, pp. 1927-1934, 2013.

[15] F. Qiao, T. Pan, J. W. Clark Jr., and O. R. Mawlawi, "A motionincorporated reconstruction method for gated PET studies," Physics in Medicine and Biology, vol. 51, no. 15, article 012, pp. 3769-3783, 2006.

[16] M. Dawood, F. Büther, N. Lang, O. Schober, and K. P. Schäfers, "Respiratory gating in positron emission tomography: a quantitative comparison of different gating schemes," Medical Physics, vol. 34, no. 7, pp. 3067-3076, 2007.

[17] B. K. Horn and B. G. Schunck, "Determining optical flow," Artificial Intelligence, vol. 17, pp. 185-203, 1981.

[18] G. Zhang, T. C. Huang, K. Lin et al., "3D optical flow method implementation for mapping of $3 \mathrm{D}$ anatomical structure contours across 4D CT data," Journal of Applied Clinical Medical Physics, vol. 9, pp. 59-69, 2008.

[19] T. Guerrero, G. Zhang, T.-C. Huang, and K.-P. Lin, "Intrathoracic tumour motion estimation from $\mathrm{CT}$ imaging using the $3 \mathrm{D}$ optical flow method," Physics in Medicine and Biology, vol. 49, no. 17, pp. 4147-4161, 2004.

[20] T. Guerrero, G. Zhang, W. Segars et al., "Elastic image mapping for $4 \mathrm{D}$ dose estimation in thoracic radiotherapy," Radiation Protection Dosimetry, vol. 115, no. 1-4, pp. 497-502, 2005.

[21] Y. E. Erdi, O. Mawlawi, S. M. Larson et al., "Segmentation of lung lesion volume by adaptive positron emission tomography image thresholding," Cancer, vol. 80, no. 12, pp. 2505-2509, 1997.

[22] T.-C. Huang and Y.-C. Wang, "Deformation effect on SUVmax changes in thoracic tumors using 4-D PET/CT scan," PLoS ONE, vol. 8, no. 3, Article ID e58886, 2013.

[23] M. Blume, A. Martinez-Möller, A. Keil, N. Navab, and M. Rafecas, "Joint reconstruction of image and motion in gated positron emission tomography," IEEE Transactions on Medical Imaging, vol. 29, no. 11, pp. 1892-1906, 2010.

[24] S. D. Wollenweber, G. Gopalakrishnan, K. Thielemans, and R. M. Manjeshwar, "Evaluation of the accuracy and robustness of a motion correction algorithm for PET using a novel phantom approach," in Proceedings of the IEEE Nuclear Science Symposium Conference, pp. 2470-2479, November 2010.

[25] S. A. Nehmeh, Y. E. Erdi, C. C. Ling et al., "Effect of respiratory gating on quantifying PET images of lung cancer," Journal of Nuclear Medicine, vol. 43, no. 7, pp. 876-881, 2002.

[26] L. Guerra, E. de Ponti, F. Elisei et al., "Respiratory gated PET/CT in a European multicentre retrospective study: added diagnostic value in detection and characterization of lung lesions," European Journal of Nuclear Medicine and Molecular Imaging, vol. 39, no. 9, pp. 1381-1390, 2012.

[27] T. C. Huang, Y. C. Wang, and C. H. Kao, “Thoracic tumor volume delineation in $4 \mathrm{D}-\mathrm{PET} / \mathrm{CT}$ by low dose interpolated CT for attenuation correction," PLoS ONE, vol. 8, Article ID e75903, 2013. 


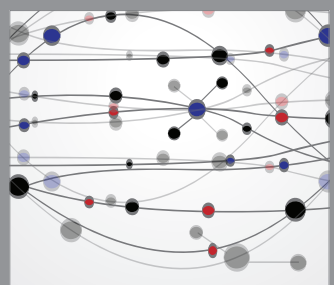

The Scientific World Journal
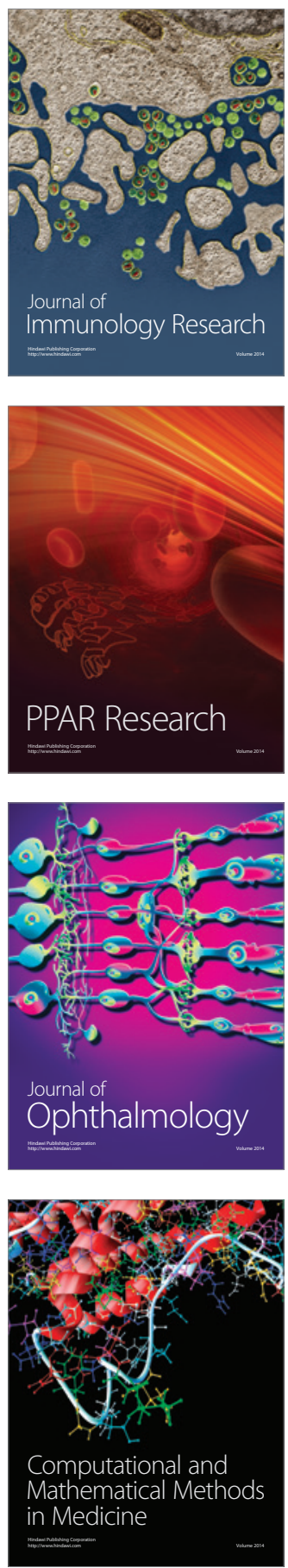

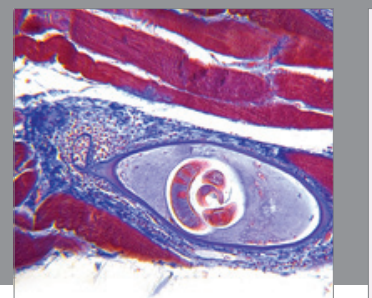

Gastroenterology

Research and Practice
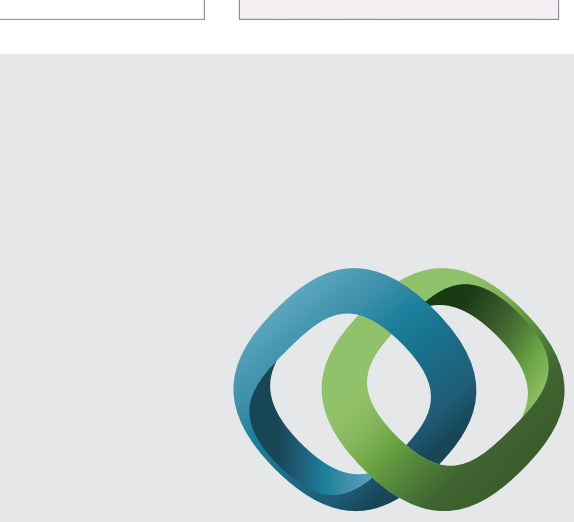

\section{Hindawi}

Submit your manuscripts at

http://www.hindawi.com
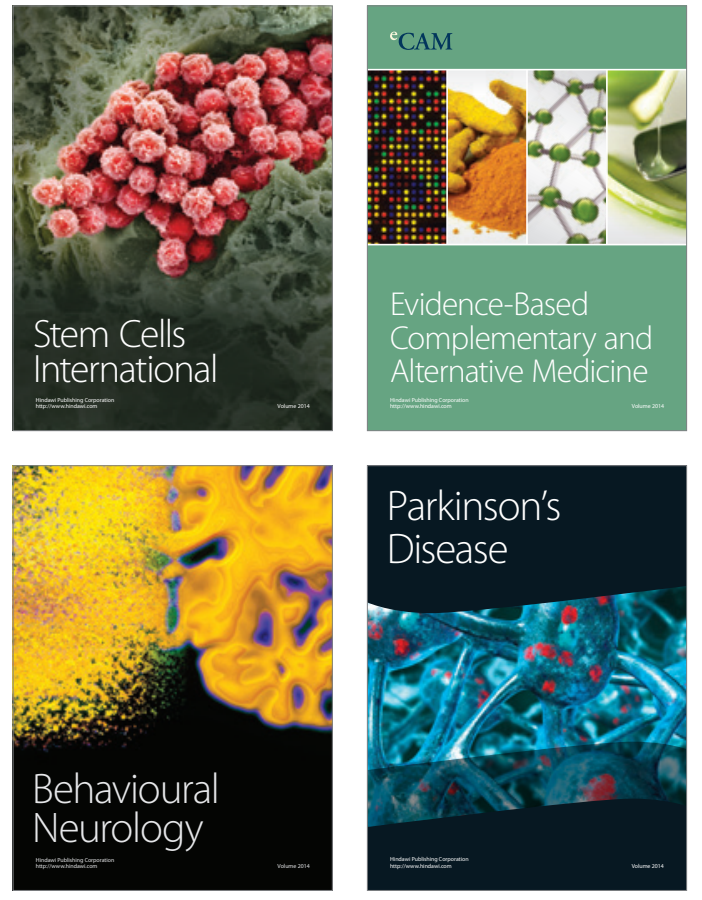
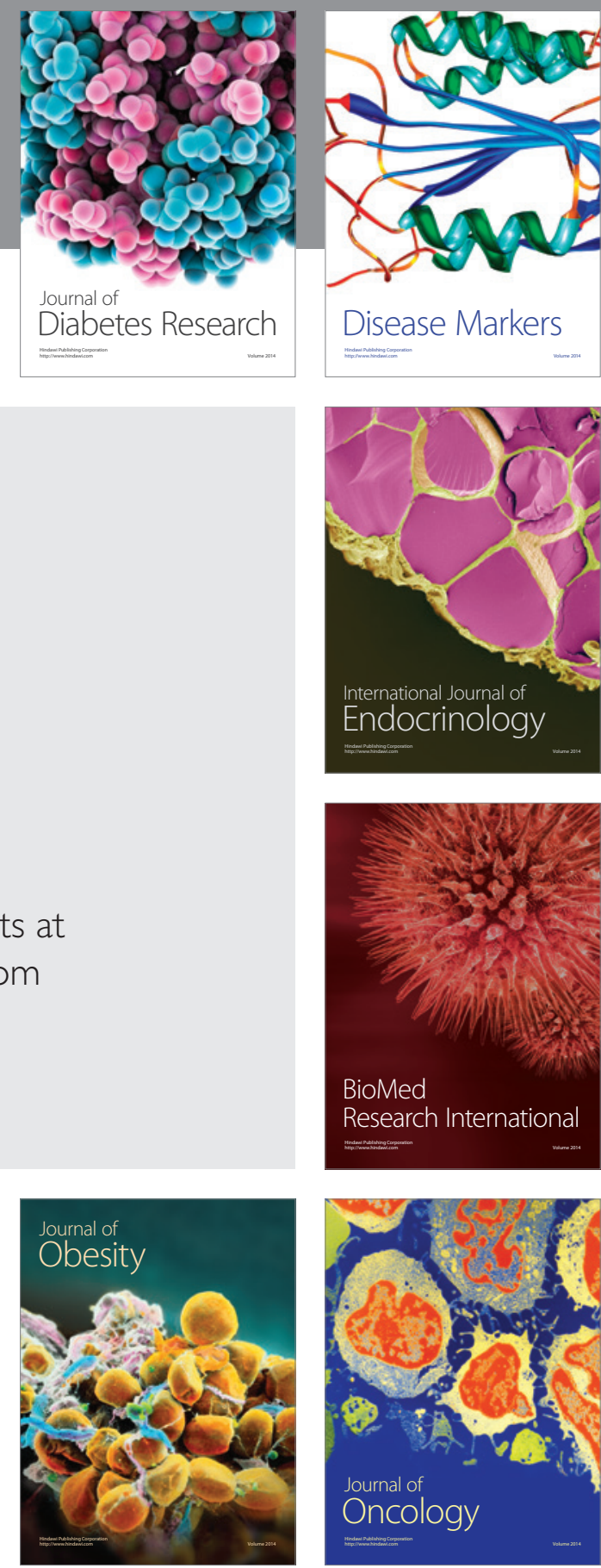

Disease Markers
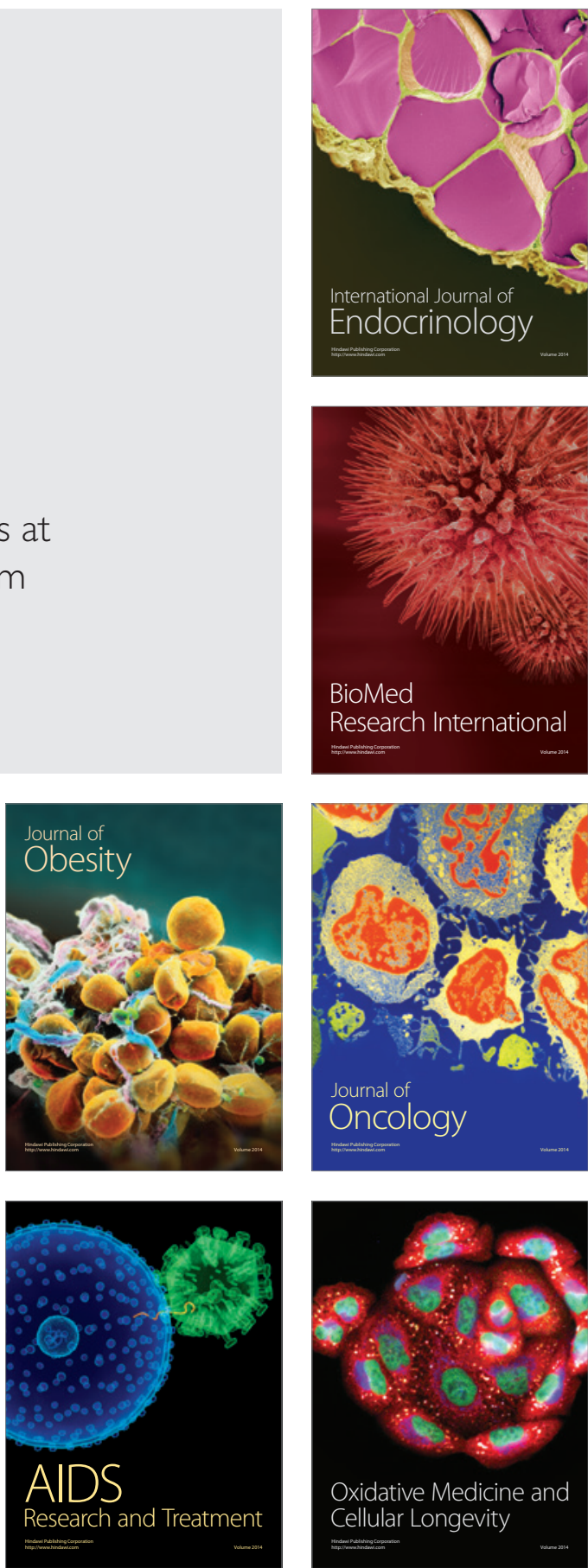